\title{
POWER QUALITY EVALUATION MODEL FOR ELECTRIC CUSTOMER BASED ON ANALYTIC HIERARCHY PROCESS
}

\author{
Buhm Lee \\ Dept. of Electrical and Semiconductor Engineering \\ Chonnam National University \\ Yeosu, South Korea \\ E-mail: buhmlee@chonnam.ac.kr \\ Kyoung Min Kim \\ Dept. of Electrical and Semiconductor Engineering \\ Chonnam National University \\ Yeosu, South Korea \\ E-mail:kkm@chonnam.ac.kr
}

\begin{abstract}
This paper presents a power quality evaluation model for electric power customer. Because every electric power customer needs high quality power, in the viewpoint of Stable Voltage, Stable Frequency, Low Harmonics, and High Reliability, they do effort to upgrade power quality. To maintain high-quality power with less-effort, this paper focuses on evaluation methodology for power quality and develops a power quality index which reflects these power quality factors by using Analytic Hierarchy Process.
\end{abstract}

Keywords: Power Quality Evaluation, Electric Customer, Reliability, Power Quality

\section{Introduction}

Power companies expend significant capital and effort to increase reliability and power quality (PQ). For quantitative assessment of PQ, indices for reliability, indices for voltage sags, and indices for harmonics, have been developed. Because these indices require all related quantities to be measured at every load point of a distribution system, measurements are not possible for nonexisting systems, i.e. systems in the planning stage. Another problem of using these individual indices is ensuing limited information from individual indices and conflict between reliability and PQ indices. Accordingly, planners have employed tradeoff analysis of individual indices when making decisions.

This paper presents a new methodology to obtain a power quality evaluation index that can assess the performance of a customer system. First, this paper classifies events into three classes: (a) Inconvenience (Power Supply), (b) Inconvenience (Clear Sinusoidal), and (c) Cost. Second, this paper proposes the use of three states to measure PQ level, such as [Ideal], [Actual], and [Possible]. Third, this paper proposes a methodology to rescale [Actual] states instead of employing one-to-one matrices. This methodology can rescale them to fit human judgment. Finally, this paper presents an Analytic Hierarchy Process(AHP) model to obtain a unified index from various indices and cost using eigenvalue analysis. By using the proposed methodology, we can obtain a unified power quality index that can show the power quality of the system, whether 
existing or non-existing. This method is especially effective for planning. For example, a decisionmaker can build expansion plan alternatives, and can choose the best plan among power quality alternatives. We applied the proposed method to real system planning, and demonstrate the effectiveness.

\section{Review of Power Quality Evaluation}

PQ of a system is quantified by following attributes: sustained reliability, momentary reliability, voltage sags, harmonics, and voltage drops. To evaluate a system, we calculate every item at each load point, and calculate system-wide indices and cost. Here, overvoltage and undervoltage which exceed $20 \%$ of nominal values and harmonics which exceed $20 \%$ of THD are considered as sustained interruptions.

We classify PQ into Inconvenience (Power Supply), Inconvenience (Clear Sinusoidal), and Cost. In Inconvenience (Power Supply) related to interruptions of power, we consider SAIFI and SAIDI as a sustained reliability, MAIFI as a momentary reliability, and $\mathrm{SARFI}_{70}$ as voltage sags. In Inconvenience (Clear Sinusoidal) related to the negative effects of the system without interruptions of power, we consider harmonics and voltage deviations. Finally, we consider costs, which can evoke system operation cost, such as harmonic aging cost, system loss cost, harmonic loss cost, and annual operation cost.

\section{Development of Ideal Analytic Hierarchy Process Model}

\subsection{3-state model}

We propose the use of three states, defined as [Ideal], [Actual], and [Possible] states in the AHP model. [Ideal] are the ideal values that customers feel as ideal, [Possible] are the possible values that customers feel as extremely challenging because of $\mathrm{PQ}$, and [Actual] are calculated values that reflect current states. As an example of voltage deviation, guess the voltage of a load point is 0 to infinitive. Even though, voltage of load point can vary 0 to infinitive, we can only load at this load point under -20 to $20[\%]$ of voltage deviation. Accordingly, 20[\%] of voltage deviation is [Possible], $0[\%]$ of is [Ideal], and $3[\%]$ is [Actual] if voltage deviation of the current state is $3[\%]$.Your study may be trying to prove something or at the very least will have a specific objective (e.g. development of a decision model for a particular problem). Make sure to list them here. The reader must be clear about the specific objectives or hypotheses in your study.

\subsection{Scaling for human sense}

In spite of the above, [Actual] state of 3-State model can reflect the current state between [Ideal] and [Possible] states, but it does not reveal the proper scale of PQ to fit human judgment. We propose a methodology that rescales [Actual] states, instead of employing one-to-one matrices. As a methodology, we normalize [Actual] state between [Ideal] and [Possible] states to overcome different standards, and apply a new non-linear scale.

\subsection{Build Ideal AHP model}

The AHP model which inherently quantifies the system performance indices is presented in a unified manner. We calculate indices and costs for 3 states, and rescale them. From rescaled 
values, we calculate an eigenvalue using a one-to-one matrix. This eigenvalue is a unified PQ index, because it can reveal the competitiveness between ideal and possible states. The smaller value indicates a better system.

\section{Application of Electric Customer}

We applied developed model for electric customer, and its procedure is as follows:

(1) PQ and its indices

We introduce system-wide indices of power quality for Alternatives.

(2) Define of States, Rescale, and one-to-one matrix

This paper introduce 3-states to apply the proposed AHP model consisting of [Ideal] - [Actual] [Possible] states. Here, Ideal states are all zero, because ideal power supply indicates no interruptions, no voltage sags, no harmonics, and no voltage fluctuation without additional operating cost. We set possible states of PQ indices considering their characteristics and arbitrarily cost. All possible states can be changed by customers and decision maker's opinion.

(3) Unified PQ index

This paper obtain eigenvalues, which are power quality evaluation index for each alternatives

\section{Conclusions}

This paper presents a customer power quality evaluation index. The contributions of the paper are:

(1) This paper proposes a new AHP model which can evaluate PQ for an electric customer system. First, authors classify three states, such as [Ideal], [Actual], and [Possible] states. Second, authors propose a rescaling methodology to fit the [Actual] state for human interpretation, rather than one-to-one matrices in AHP. Third, authors present an AHP model to obtain unified index using eigenvalue analysis from various indices and cost. By using this model, obtained Power Quality evaluation index can show the PQ of the electric customer system, regardless of whether it exists.

(2) By using a customer power quality index that includes cost, the decision maker can select the most effective alternative without a pareto-optimal solution.

(3) This paper applies this methodology to a relatively large distribution system under expansion, and show the usefulness in planning is confirmed. The study case demonstrates the process of selecting the best system in terms of improving power quality at low cost.

\section{Key References}

Saaty, T.L. (1996). The Analytic Network Process: Decision Making with Dependence and Feedback. Pittsburgh, PA: RWS Publications.

Ilic, M.D. et al. (1996). Hierarchical Power Systems Control - Its Value in a Changing Industry. Springer. 
IJAHP Article: Mu, Saaty/A Style Guide for Paper Proposals To Be Submitted to the International Symposium of the Analytic Hierarchy Process 2014, Washington D.C., U.S.A.

Forman, Ernest. Decision by Objectives. http://mdm.gwu.edu/forman

IEEE (1992). IEEE Recommended Practices and Requirements for Harmonic Control in Electrical PowerSystems. IEEE Std 519”, 1992

IEEE (1995). IEEE Recommended Practice for Monitoring Electric Power Quality. IEEE Std 1159”

IEEE (2001). IEEE Guide for Electric Power Distribution Reliability Indices. IEEE Std $1366 ", 2001$

Math, H. J. et al. (2003). Voltage-Sag Indices - Recent Developments in IEEE P1564 Task Force. pp34-41 